\title{
Encuesta de síntomas SNOT-20 para rinitis alérgica y rinosinusitis: validación en Chile
}

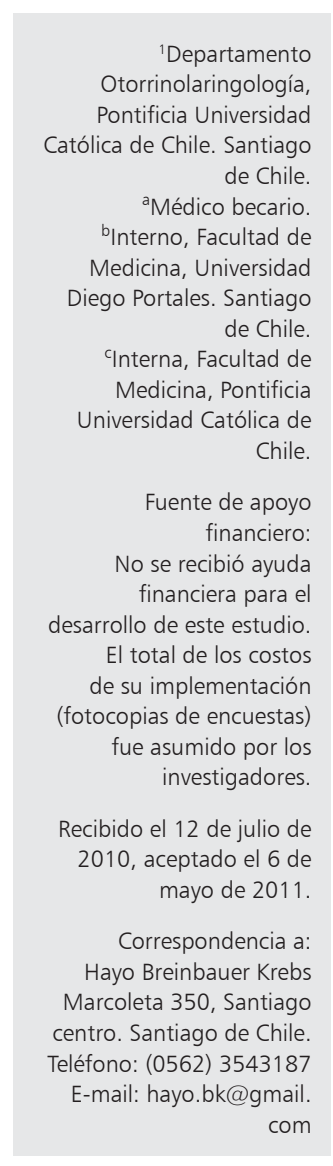

\author{
HAYO BREINBAUER ${ }^{1, a}$, CECILIA VARELA ${ }^{\mathrm{b}}$, MAURICIO NÚÑEZ, \\ SOLEDAD UGARTE ${ }^{c}$, RAÚL GARFIAS ${ }^{1, a}$, XIMENA FONSECA ${ }^{1}$
}

\section{Validation of the sino-nasal outcome test in Chile}

Background: Allergic rhinitis and sinusitis are common diseases that affect quality of life of pediatric and adult patients. Aim: To adapt and validate the Sino-Nasal Outcome Test 20 indicators (SNOT-20) survey in Chilean subjects. Material and Methods: In a psychometric validation study, an adapted version of the SNOT-20 adapted version was applied to 181 volunteers on two different occasions, three weeks apart. Feasibility (response time and perceived difficulty), reliability (internal consistency and test-retest) and validity (concurrent validity, correlating results with an independent instrument; predictive validity assessing its sensitivity to detect changes and discriminant validity, assessing receiver operating characteristic (ROC) curves), were assessed. An exploratory factor analysis was performed to confirm the presence of previously described underlying constructs. Results: Eighty percent of participants considered SNOT-20 easy to complete. This task was completed in less than 5 minutes. Cronbach's alpha was 0.946. Temporal stability on a healthy subgroup was strong with an intraclass correlation coefficient of 0.92. SNOT-20 had a correlation coefficient of 0.82 with an independent instrument and its score significantly decreased after 3 weeks of treatment $(p=0.003)$. The area under ROC curve was 0.895 . Factor analysis identified four principal components which possessed identical structure as previously described. Conclusions: SNOT-20 survey was valid, reliable and easy to implement among Chilean subjects.

(Rev Med Chile 2011; 139: 886-895).

Key words: Assessment, patient outcomes; Rhinitis, allergic, perennial; Sinusitis.
L a rinitis alérgica y la rinosinusitis crónica se encuentras dentro de las patologías crónicas más frecuentes tanto en población pediátrica como adulta ${ }^{1}$, presentando ambas entidades sintomatología similar y sobrepuesta, al punto de considerarse por algunos autores como extremos de una gama de patologías inflamatorias de la vía respiratoria superior ${ }^{2-4}$.

La presencia de síntomas nasosinusales (rinorrea, estornudos, congestión nasal, descarga posterior, algia facial, otalgia, etc.) alcanza $45,7 \%$ en escolares del gran Santiago ${ }^{5}$, con $21,9 \%$ de diagnóstico definitivo para rinitis alérgica en este grupo ${ }^{6}$.

En población adulta, 1,3\% de todas las consultas médicas en EE.UU. de Norteamérica responden a cuadros rinosinusales, y se estima una prevalencia de $15,5 \%$ de rinosinusitis crónica en la población general norteamericana ${ }^{7,8}$.

Estas patologías tienen alto impacto en la calidad de vida de los pacientes que las sufren, alcanzando peores indicadores que diabetes mellitus, artritis reumatoide o migraña ${ }^{9,10}$, no sólo en relación a síntomas locales como rinorrea, algia 
facial o hiposmia, sino secundario a alteraciones del sueño, deterioro de la autoestima social y fatiga general $^{11}$.

En consecuencia se aconseja contar con instrumentos que permitan registrar intensidad percibida de síntomas y su impacto en calidad de vida, tanto en investigación, como en la práctica clínica cotidiana ${ }^{2,12}$

El desarrollo de dichos instrumentos ha atravesado diversas etapas, comenzando con acuciosos cuestionarios como RhinoQOL y RSOM-31, ambos extensos y de compleja interpretación. Seleccionando los indicadores más representativos y sensibles de estas encuestas se diseñó SNOT-20, siendo ampliamente utilizada y recomendada ${ }^{4,12-15}$.

El Sino-Nasal Outcome Test de 20 indicadores (SNOT-20) es un cuestionario de autoaplicación desarrollada por Piccirillo en inglés ${ }^{13}$ y ha sido adaptada al alemán ${ }^{16}$, chino ${ }^{17}$ y japonés ${ }^{18}$. Cada indicador es de tipo Lickert de 6 niveles (Tabla 1-a) y evalúa la intensidad de un síntoma o el impacto en algún aspecto de calidad de vida. Adicionalmente el paciente puede elegir los 5 indicadores que le parezcan más molestos y que le gustaría cambiar con un eventual tratamiento (indicadores-objetivo).

Para interpretar el instrumento se utiliza la media de los 20 indicadores (valor entre 0 y 5 ). Una diferencia de 0,8 puntos entre grupos es considerada como clínicamente significativa ${ }^{13}$. Es necesario enfatizar que SNOT-20 no está diseñada como herramienta diagnóstica (no es adecuado utilizar puntajes de corte buscando establecer existencia de rinitis alérgica o rinosinusitis). Su propósito es evaluar intensidad de síntomas e impacto en calidad de vida, con el fin de comparar efectividad de tratamientos sobre patologías nasosinusales ${ }^{2}$.

$\mathrm{Su}$ interpretación y significado clínico ha sido enriquecida al incorporar el análisis de 4 sub-escalas: síntomas rinológicos, síntomas óticofaciales, función del sueño y función psicológica ${ }^{14}$ (Tabla 1).

Este cuestionario ha sido ampliamente utilizado, principalmente en la evaluación de efectividad de distintos tratamientos, incluyendo respuesta a budesonida y otros corticoides inhalatorios en rinosinusitis crónica y rinitis alérgica ${ }^{4,19}$, antibioterapia como moxifloxacino y macrolidos en rinosinusitis aguda y crónica $\mathrm{ca}^{20-22}$, distintas variantes de tratamiento quirúrgico ${ }^{23-25}$, así como el impacto de tabaquismo sobre sintomatología rinosinusal ${ }^{26}$. También ha servido para indagar la relación entre patología de vía aérea superior e inferior bajo el concepto de vía aérea unificada ${ }^{27,28}$. En todos estos estudios SNOT-20 ha demostrado ser sensible en encontrar diferencias significativas entre los distintos grupos estudiados.

El presente estudio presenta una adaptación en español de SNOT-20, evaluando su validez y confiabilidad en un contexto chileno, esperando aportar un instrumento útil para las distintas especialidades relacionadas con patología de la vía aérea superior.

\section{Material y Método}

\section{Estudio de validación psicométrica en tres etapas}

Primera etapa: Se adaptó SNOT-20 al español mediante traducción y retro-traducción. Veintiséis voluntarios realizaron aplicación piloto, donde se midió tiempo necesario para completar el instrumento adaptado.

Segunda etapa: Se aplicó SNOT-20 traducida a una muestra tanto de pacientes con sospecha de patología nasosinusal como de voluntarios sanos (previo consentimiento informado). Al momento de la aplicación, la encuesta fue entregada con la indicación de completarla utilizando todo el tiempo que fuese necesario, sin realizar preguntas a los investigadores.

Se complementó SNOT-20 con algunos indicadores tipo Lickert explorando intensidad subjetiva tanto del problema nasosinusal como un todo y separado en los cuatro dominios descritos en sus subescalas $^{14}$ (Tabla2) Se indago además pertinencia y grado de dificultad de la encuesta según los mismos encuestados.

Tercera etapa: Se intentó reaplicar el instrumento vía contacto telefónico (autorizado en consentimiento previo) en ambos grupos de la muestra luego de 3 semanas, registrando si existió cualquier tipo de cambio en su esquema de tratamiento durante este intervalo.

El grupo de pacientes con sospecha de patología nasosinusal fue reclutado en la consulta del Departamento de Otorrinolaringología de la Pontificia Universidad Católica de Chile por residentes y médicos de dicho servicio. Como criterio de inclusión se consideró a todo paciente que consultara espontáneamente por síntomas nasosinusales, sin importar el diagnóstico clínico elaborado por el médico. 


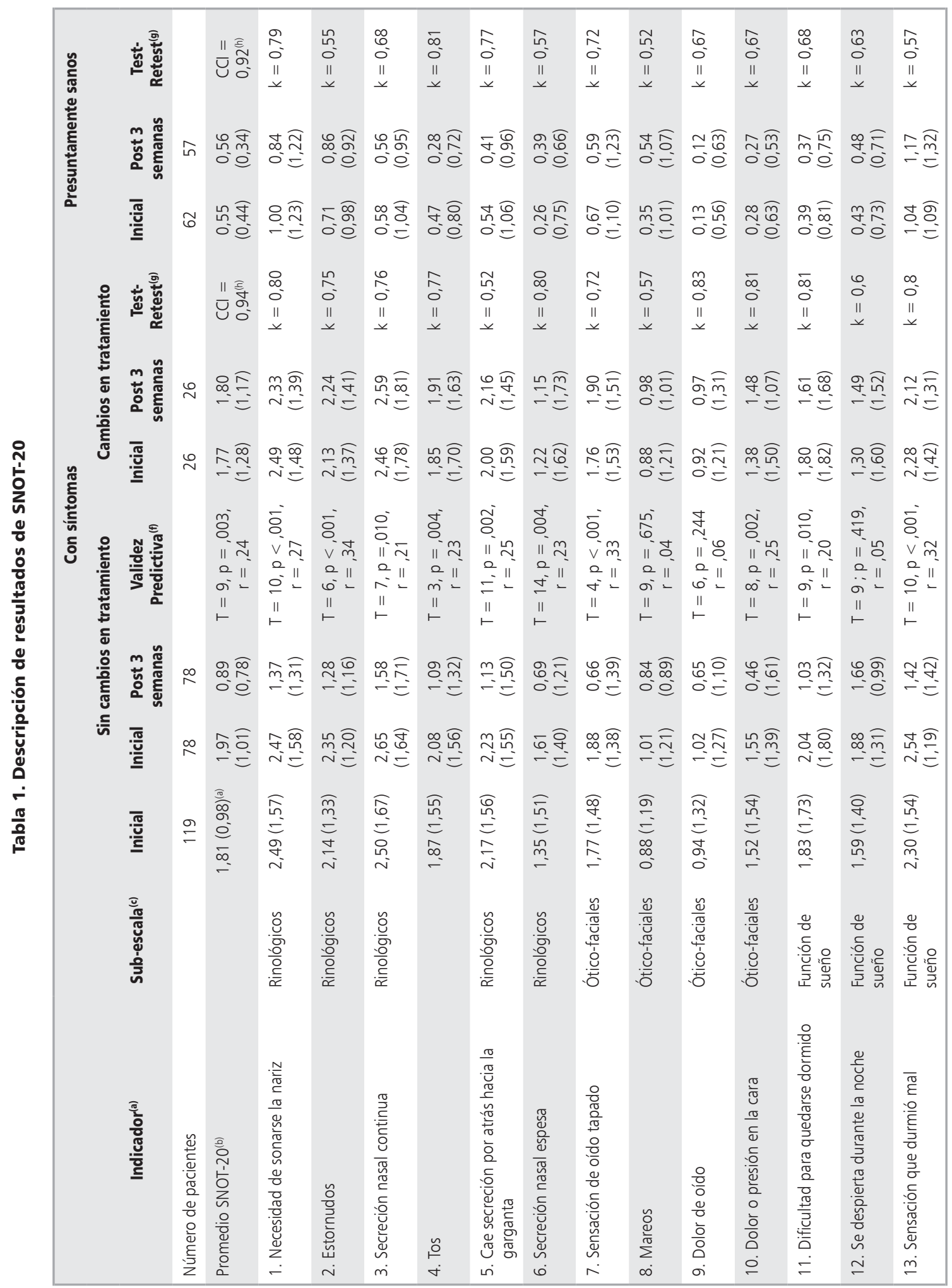




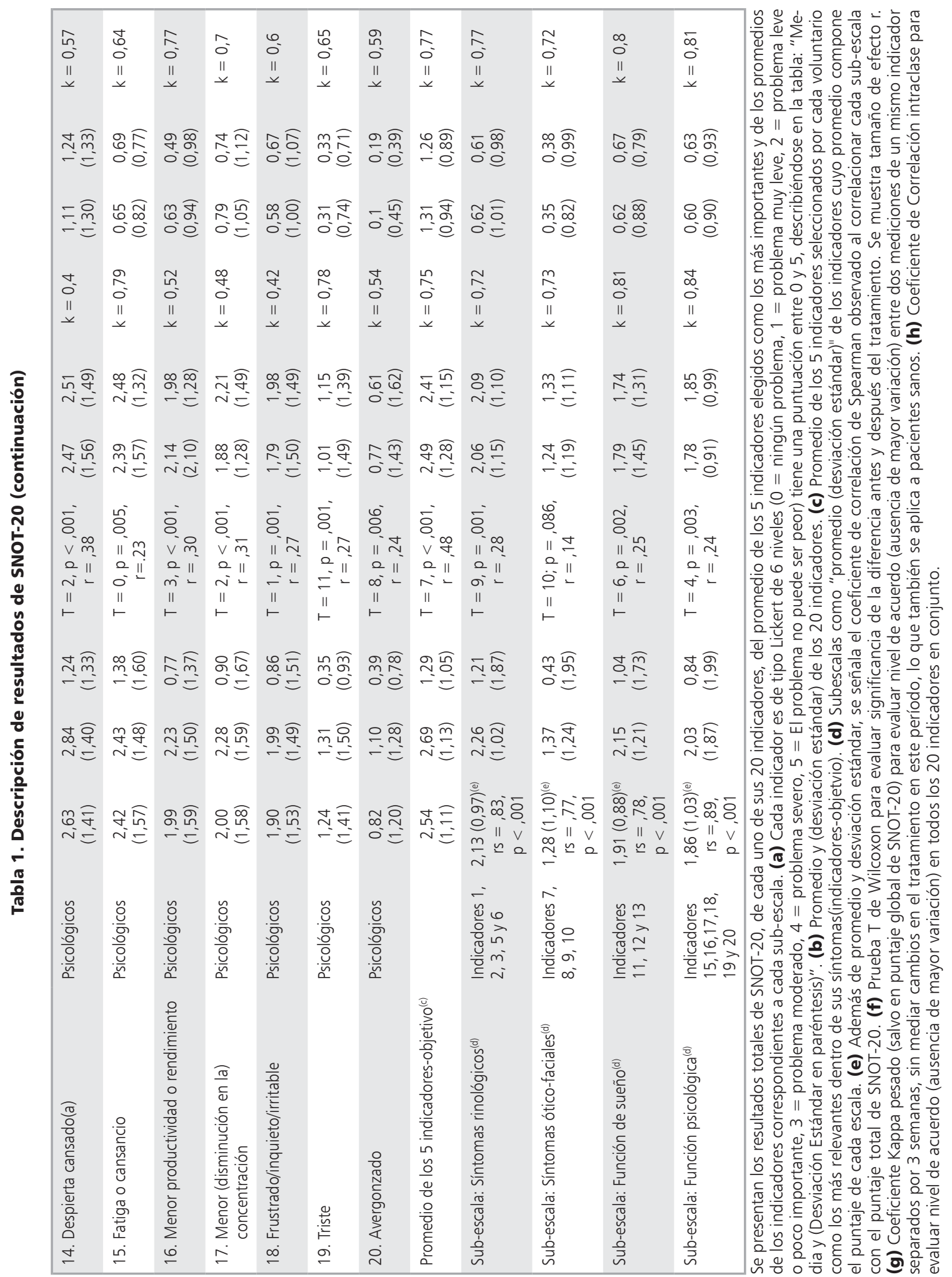


Los voluntarios sanos fueron reclutados a partir de estudiantes de medicina de primer a tercer año y sus familiares, considerando como criterio de exclusión el tener una patología nasosinusal conocida.

Considerando la dispersión encontrada en estudios semejantes ${ }^{13,16-18}$, poder estadístico de $90 \%$, error tipo alfa de $5 \%$ para dos colas, y una diferencia clínicamente significativa de al menos 0,8 puntos en resultado de SNOT-20, se estimó un tamaño muestral necesario de 124 individuos.

En el análisis, la factibilidad del instrumento se evaluó en base al tiempo necesario para completarlo y la apreciación de los voluntarios sobre su dificultad. Para validez de apariencia se usó la revisión bibliográfica descrita y la opinión de los encuestados sobre pertinencia del instrumento. Para confiabilidad se evaluaron consistencia interna y estabilidad temporal (test-retest) en los grupos sin cambios en su tratamiento (incluye sanos). Se realizaron múltiples procedimientos para validez de constructo: validez concurrente mediante la comparación de los resultados globales y por escalas de SNOT-20 con los indicadores complementarios descritos, análisis factorial exploratorio buscando corroborar la existencia de sub-escalas descritas, y validez predictiva al evaluar cambios luego de 3 semanas de tratamiento. Se realizó además estudio de validez discriminante mediante curvas ROC, ocupando como discriminante el pertenecer al grupo de pacientes sanos o consultantes por sintomatología nasosinusal.

Dada la distribución no-normal encontrada en el grupo con sintomatología nasosinusal y el carácter ordinal del instrumento, se prefirieron pruebas no paramétricas. Se utilizó SPSS 17.0 para realizar pruebas chi cuadrado sin corrección de Yates, U de Mann-Whitney, T de Wilcoxon, coeficientes de correlación Rho de Spearman, pesado Kappa, alfa de Cronbach, de correlación intraclase, y análisis factorial exploratorio, según correspondiese.

\section{Resultados}

Dos otorrinolaringólogos independientes realizaron la traducción al español privilegiando términos dentro del lenguaje cotidiano chileno. Ambas versiones tenían mínimas diferencias, y con un tercer especialista se consensuó una versión final. Dentro de los indicadores difícil traducción destacó "Dolor o presión en la cara", considerando que muchos pacientes en nuestro contexto asocian este síntoma a un más general "dolor de cabeza". Se planteó ampliar este indicador incorporando una dimensión más cercana a cefalea. Sin embargo, un indicador semejante fue descartado en el proceso original de SNOT-20 desde RSOM-31, 13,13 , justamente por ser demasiado amplio e incluir dimensiones "neurológicas" y "tensionales". En base a estos criterios, se decidió dejar el indicador apuntando a una dimensión más "facial" lo que se apoya además en la sub-escala descrita ${ }^{14}$. Se corrigieron detalles mínimos de redacción en base a aplicación piloto en 26 voluntarios.

Esta versión fue retro-traducida al inglés por otros dos médicos independientes. Ambas versiones coincidían casi perfectamente con el instrumento original, con lo que se aceptó la versión final para someterla al estudio de validación.

Se reclutaron 181 voluntarios, 119 dentro del grupo que consultó espontáneamente por síntomas nasosinusales y 62 supuestamente sanos. En la re-aplicación telefónica se logró contactar a 161 voluntarios, 78 de los cuales habían modificado su tratamiento para su patología nasosinusal, mientras que 26 individuos no habían alterado su manejo y 57 eran pacientes sanos. La distribución del puntaje SNOT-20 se comportó de forma nonormal en el grupo con síntomas nasosinusales $($ Kolmogorov-Smirnov D $(119)=, 155 ; \mathrm{p}<, 001)$.

La edad promedio fue 29,9 años con desviación estándar (DE) de 14,6 años. 54,6\% fue de género femenino. No existieron diferencias significativas en términos de edad o género entre grupos "sintomático" y "sano" (edad: $\mathrm{U}=90,5 ; \mathrm{p}=0,314$; género: $\left.\chi^{2}(1)=0,034 ; p=0,854\right)$ o entre "con cambios en tratamiento" y "sin cambios en tratamiento" (edad: $U=98,5 ; \mathrm{p}=0$, 832; género: $\chi^{2}$ $(1)=0,210 ; p=0,646)$.

Los cinco indicadores más frecuentemente elegidos como indicadores-objetivo fueron "Necesidad de Sonarse la Nariz" (43\% de los voluntarios), "Secreción Nasal Continua”(38\%), "Cae secreción por atrás hacia la garganta”(34\%), "Sensación de haber dormido mal"(32\%) y "Despertar cansado"(27\%).

\section{Factibilidad}

Los 181 encuestados respondieron el 100\% de los 20 indicadores. 78,8\% seleccionó 5 indi- 
Encuesta sobre rinitis alérgica y rinosinusitis - $\mathrm{H}$. Breinbauer et al

cadores-objetivo mientras que el resto omitió este aspecto del instrumento. 79,5\% consideró el instrumento fácil o muy fácil de contestar y ninguno lo consideró difícil de responder. El tiempo promedio necesario para completar SNOT-20 fue 3 minutos y 21 segundos (DE 46 segundos).

\section{Validez de apariencia}

SNOT-20 ha sido validada y recomendada ampliamente por numerosos comités de expertos $^{2,4,8,12-15}$. 76,6\% del grupo "con síntomas" (opinión de los involucrados) consideró el instrumento como pertinente o muy pertinente.

\section{Confiabilidad}

La consistencia interna mostró un coeficiente alfa de Cronbach $=, 946$. En cuanto a estabilidad temporal (test-retest), considerando dos evalua- ciones separadas por 3 semanas en los encuestados sanos o sin cambios en su tratamiento, el coeficiente $\kappa$-pesado fue $r=0,52-0,81$ para cada indicador y el coeficiente de correlación intraclase fue CCI $=0,92-0,94$ para los 20 indicadores en conjunto (Tabla 1).

\section{Procedimientos para validez de constructo}

Validez concurrente. Al comparar el indicador independiente para intensidad global del problema nasosinusales con el puntaje SNOT-20 se obtiene $r_{s}=-82 ; \mathrm{p}<0,001 ; \mathrm{y}_{\mathrm{s}}=0,79 ; \mathrm{p}<0,001$ con el promedio de los 5 indicadores-objetivo (Tabla 2 ).

Análisis factorial exploratorio. Se realizó análisis de componentes principales con rotación oblicua (Promax) dada interdependencia de los factores subyacentes. La medida de adecuación del tamaño

Tabla 2. Indicadores anexos a SNOT-20 para validez concurrente

\begin{tabular}{|c|c|c|c|}
\hline \multicolumn{2}{|c|}{ Indicadores Subjetivos ${ }^{(a)}$} & \multirow{3}{*}{$\begin{array}{c}\text { Promedio y } \\
\text { (desviación estándar) } \\
\text { para cada indicador } \\
3,10(\mathrm{DE} 1,64)\end{array}$} & \multirow{3}{*}{$\begin{array}{l}\text { Correlación de Spearman con } \\
\text { escala respectiva }{ }^{(\mathbf{b})} \\
r_{s}=0,82 ; p<0,001 \\
\text { Correlación con resultado final de } \\
\text { SNOT-20 } \\
r_{s}=0,79 ; p<0,001 \\
\text { Correlación con promedio de los } 5 \\
\text { indicadores seleccionados como los } \\
\text { más importantes en cada voluntario }\end{array}$} \\
\hline $\begin{array}{l}\text { Impacto global } \\
\text { de molestias } \\
\text { naso-sinusales }\end{array}$ & $\begin{array}{l}\text { Considerando en conjunto todos } \\
\text { sus síntomas, ¿qué importancia a } \\
\text { su problema naso-sinusal, rinitis }\end{array}$ & & \\
\hline & alérgica o de rinosinusitis? & & \\
\hline \multirow[t]{4}{*}{$\begin{array}{l}\text { Impacto por } \\
\text { sub-escalas }\end{array}$} & $\begin{array}{l}\text { Problemas con secreciones y } \\
\text { mucosidades nasales. }\end{array}$ & 2,73 (DE 1.67) & $\begin{array}{l}r_{s}=0,78 ; p<0,001 \\
\text { Correlación con puntuación subescala } \\
\text { "síntomas rinológicos" }\end{array}$ \\
\hline & $\begin{array}{l}\text { Molestias en oídos, cara y cabeza } \\
\text { en general }\end{array}$ & 2,80 (DE 1,59) & $\begin{array}{l}r_{s}=0,65 ; p<0,001 \\
\text { Correlación con puntuación subescala } \\
\text { "síntomas Ótico-faciales" }\end{array}$ \\
\hline & Problemas para dormir & $2,52(\mathrm{DE} 1,50)$ & $\begin{array}{l}r_{s}=0,78 ; p<0,001 \\
\text { Correlación con puntuación subescala } \\
\text { "función de sueño" }\end{array}$ \\
\hline & $\begin{array}{l}\text { Problemas psicológicos (autoesti- } \\
\text { ma, problemas al relacionarse con } \\
\text { otras personas, etc) }\end{array}$ & $1,73(\mathrm{DE} 1,38)$ & $\begin{array}{l}r_{s}=0,54 ; p<0,001 \\
\text { Correlación con puntuación subescala } \\
\text { "función psicológica" }\end{array}$ \\
\hline
\end{tabular}

Asociado a la aplicación inicial de SNOT-20 se evaluaron 5 indicadores subjetivos buscando cubrir la dimensión global del instrumento, y las dimensiones correspondientes a las sub-escalas previamente descritas ${ }^{14}$.(a) Cada una de los 5 indicadores subjetivos, fueron evaluados como indicador tipo Lickert de 7 niveles, considerando la siguiente pauta: Ningún problema = 1 , Problema insignificante $=2$, Problema leve $=3$, Problema moderado $=4$, Problema severo $=5$, Problema muy severo $=6$, Peor problema posible $=7$. (b) Se evaluó el grado de correlación entre el resultado de cada uno de estos indicadores subjetivos con los resultados de SNOT-20 respectivos (resultado final, media de los 5 indicadores seleccionados como los más importantes y medias de cada sub-escala) a las dimensiones que los indicadores subjetivos buscaban evaluar. Para ello se utilizó correlación de Spearman. 
muestral de Kaiser-Meyer-Olkin fue ,88 (catalogado como "muy bueno" 29 ), los valores KaiserMeyer-Olkin para cada indicador fueron mayores a 0,63 (superando el 0,5 definido como límite aceptable $^{29}$ ) y el test de esfericidad de Bartlett fue $\chi^{2}(190)=2881,57 ; p<0,001$, todo lo indica que el tamaño muestral fue adecuado y que el análisis factorial es confiable.

Previo a la rotación, se analizaron los componentes asignando eigenvalues (Tabla 3). En base al criterio de Kaiser $^{29}$ (seleccionar componentes con eigenvalues superiores a 1) y el punto de inflexión

Tabla 3. Análisis Factorial exploratorio

\begin{tabular}{|c|c|c|c|c|}
\hline \multirow[t]{2}{*}{ Análisis factorial exploratorio } & \multicolumn{4}{|c|}{$\begin{array}{l}\text { Factores luego de la extracción (se identifican con los } \\
\text { nombres de sub-escalas definidos previamente }{ }^{14} \text { ) }\end{array}$} \\
\hline & $\begin{array}{l}\text { Síntomas } \\
\text { Rinológicos }\end{array}$ & $\begin{array}{l}\text { Función } \\
\text { Psicológica }\end{array}$ & $\begin{array}{l}\text { Función de } \\
\text { Sueño }\end{array}$ & $\begin{array}{l}\text { Síntomas } \\
\text { Ótico-Faciales }\end{array}$ \\
\hline Eigenvalues previos a la rotación & 9,966 & 2,123 & 1,264 & 1,088 \\
\hline Porcentaje de la varianza previos a la rotación & $48,82 \%$ & $10,61 \%$ & $6,32 \%$ & $5,43 \%$ \\
\hline $\begin{array}{l}\text { Cuadrados de las cargas de los componentes } \\
\text { luego de la rotación }\end{array}$ & 7,604 & 8,125 & 6,362 & 5,389 \\
\hline \multirow[t]{2}{*}{ Porcentaje de la varianza luego de la rotación } & $19,42 \%$ & $21,17 \%$ & $16,58 \%$ & $14,02 \%$ \\
\hline & \multicolumn{4}{|c|}{$\begin{array}{c}\text { Carga del componente de cada indicador sobre cada factor } \\
\text { (subescala) luego de la rotación }\end{array}$} \\
\hline 1. Necesidad de sonarse la nariz & 0,954 & $-0,037$ & $-0,069$ & $-0,104$ \\
\hline 2. Estornudos & 1,018 & $-0,074$ & $-0,084$ & $-0,141$ \\
\hline 3. Secreción nasal continua & 0,837 & 0,091 & $-0,012$ & $-0,034$ \\
\hline 4. Tos & 0,367 & $-0,164$ & 0,145 & 0,288 \\
\hline 5. Cae secreción por atrás hacia la garganta & 0,817 & $-0,115$ & 0,152 & 0,022 \\
\hline 6. Secreción nasal espesa & 0,616 & 0,255 & $-0,103$ & 0,158 \\
\hline 7. Sensación de oído tapado & 0,107 & 0,089 & $-0,146$ & 0,610 \\
\hline 8. Mareos & $-0,241$ & $-0,005$ & 0,112 & 0,911 \\
\hline 9. Dolor de oído & 0,111 & $-0,044$ & 0,154 & 0,568 \\
\hline 10. Dolor o presión en la cara & 0,283 & 0,238 & 0,050 & 0,548 \\
\hline 11. Dificultad para quedarse dormido & $-0,117$ & 0,150 & 0,743 & 0,185 \\
\hline 12. Se despierta durante la noche & 0,075 & $-0,255$ & 0,921 & 0,117 \\
\hline 13. Sensación que durmió mal & $-0,017$ & 0,261 & 0,796 & $-0,180$ \\
\hline 14. Despierta cansado(a) & 0,026 & 0,372 & 0,445 & $-0,137$ \\
\hline 15. Fatiga o cansancio & 0,347 & 0,511 & 0,197 & 0,100 \\
\hline 16. Menor productividad o rendimiento & 0,075 & 0,882 & $-0,051$ & $-0,016$ \\
\hline 17. Menor (disminución en la) concentración & $-0,008$ & 0,748 & $-0,064$ & 0,236 \\
\hline 18. Frustrado/inquieto/irritable & $-0,083$ & 0,943 & 0,058 & $-0,070$ \\
\hline 19. Triste & $-0,035$ & 0,916 & 0,008 & $-0,103$ \\
\hline 20. Avergonzado & $-0,034$ & 0,726 & $-0,161$ & 0,286 \\
\hline
\end{tabular}

Luego de extracción mediante método de componentes principales (utilizando criterio de Kaiser) e interpretación de punto de inflexión en gráfico de sedimentación y rotación oblicua (Promax) con normalización Kaiser, se conservaron para el análisis 4 componentes. Se decidió nombrar dichos factores según escalas previamente descritas dada similitud de su distribución ${ }^{14}$. Se describen eigenvalues y distribución porcentual de la varianza para cada factor antes y después de la rotación. Dentro de la descripción del grado de carga de cada factor sobre cada indicador, se resaltan en negrita aquellas mayores a 0,5 lo que coincide con los indicadores previamente identificados ${ }^{14}$ como los elementos constitutivos de cada sub-escala. 
Tabla 4. Correlaciones entre factores seleccionados

\begin{tabular}{|lcccc|}
\hline \multicolumn{4}{|c}{$\begin{array}{c}\text { Matriz de correlaciones de componentes } \\
\text { (factores seleccionados luego de extracción, que corresponden con las sub-escalas) }\end{array}$} \\
Componente-factores & $\begin{array}{c}\text { Síntomas } \\
\text { rinológicos }\end{array}$ & $\begin{array}{c}\text { Función } \\
\text { psicológica }\end{array}$ & $\begin{array}{c}\text { Función } \\
\text { de sueño }\end{array}$ & $\begin{array}{c}\text { Síntomas } \\
\text { ótico-faciales }\end{array}$ \\
\hline Síntomas rinológicos & 1,000 & 0,563 & 0,510 & 0,463 \\
\hline Función psicológica & 0,563 & 1,000 & 0,623 & 0,535 \\
\hline Función de sueño & 0,510 & 0,623 & 1,000 & 0,452 \\
\hline Síntomas ótico-faciales & 0,463 & 0,535 & 0,452 & 1,000 \\
\hline
\end{tabular}

Se aprecian las correlaciones de Spearman resultantes entre los cuatro componentes restantes luego de la extracción en el análisis factorial exploratorio. Destaca que las correlaciones de la escala "función psicológica" con los otros tres componentes son las mayores y siempre superiores a 0,5.

de eigenvalues en estudio de sedimentación, se seleccionaron 4 de 20 componentes para el análisis final, que explican $71,2 \%$ de la varianza.

El grado de carga de cada componente sobre

\begin{tabular}{|c|c|c|}
\hline \multicolumn{3}{|c|}{ Curva ROC } \\
\hline
\end{tabular}

Figura 1. Curva ROC. Considerando como discriminante el pertenecer al grupo con síntomas nasosinusales vs pertenecer al grupo sano. los indicadores de SNOT-20 se aprecian en la Tabla 3 , y corresponden adecuadamente a la estructura de las cuatro escalas descritas previamente ${ }^{14}$ (se describen estos componentes con los nombres asignados para las sub-escalas). Las correlaciones entre los cuatro componentes se aprecian en la Tabla 4, y describen una alta relación entre sí. Al comparar los indicadores independientes que exploran el dominio de cada sub-escala con la puntuación de cada sub-escala de SNOT-20, se encontraron $r_{s}=0,54-0,78 ; p<0,001$ (Tabla 2).

Validez predictiva. La variación del puntaje de SNOT-20 luego de 3 semanas bajo algún tipo de tratamiento fue -0,98 $(\mathrm{T}=9, \mathrm{z}=-2,984 ; \mathrm{p}<0,003$; $r=0,24$ - Tabla 1). Esta diferencia fue mayor $(-1,4)$ al evaluar el cambio en los 5 indicadores-objetivo $(\mathrm{T}=7, \mathrm{z}=-6,031 ; \mathrm{p}<0,001, \mathrm{r}=0,48)$.

Validez discriminante. El análisis de curva ROC (Figura 1) obtuvo área bajo la curva de 0,895 (intervalo 0,852 - 0,939 con 95\% de confianza).

\section{Discusión}

El comportamiento general de SNOT-20 en nuestra muestra guarda gran similitud con experiencias previas en otros países. Al igual que en el estudio original de Piccirillo, las mayores puntuación estuvieron en los indicadores relativos a secreciones y molestias nasales (indicadores 1, 3, 5), y cansancio y fatiga general (indicadores 15 y 16), con desviaciones estándar muy semejantes ${ }^{13}$.

El completar el instrumento fue fácil y rápido 
para los voluntarios, con un tiempo requerido menor a 5 minutos, mostrándose como una herramienta de alta factibilidad. Sólo la tarea opcional de seleccionar los indicadores más relevantes dentro de la propia sintomatología resulto desafiante con $21,2 \%$ de omisión.

SNOT-20 mostró gran confiabilidad (distintas mediciones obtienen siempre respuestas similares) con valores de consistencia interna y estabilidad en el tiempo superiores a 0,92 considerando la encuesta completa, y superiores a 0,52 al analizar cada indicador por separado.

Los distintas procedimientos para validez de constructo apoyan fuertemente la validez del instrumento en nuestro contexto (mediría lo que pretende medir).

Al comparar el puntaje de SNOT-20 con otro instrumento que explora globalmente el mismo fenómeno (validez concurrente), la correlación fue muy alta $(0,82$, Tabla 2$)$. El carácter subjetivo de este instrumento paralelo cobra mayor sentido bajo el concepto aceptado en la literatura de que los hallazgos al examen físico e imagenológicos no guardan buena relación con la experiencia subjetiva de los pacientes sobre su sintomatología 2 .

El análisis factorial corrobora fuertemente la validez de esta adaptación. Haber obtenido exactamente las mismas cuatro sub-escalas (no se añadió o eliminó ningún indicador a las escalas ${ }^{14}$ ) (Tabla 3) habla del sólido comportamiento de los constructos subyacentes al cuestionario a pesar de ser aplicado en distintos contextos. Cada sub-escala se correlaciona importantemente con instrumentos independientes que exploran los mismos dominios (0,54-0,79 - Tabla 2). Estos hallazgos sugieren que SNOT-20 evalúa un fenómeno compuesto por estos dominios, de forma relativamente independiente de factores culturales o personales.

El instrumento confirmó su sensibilidad a cambios en el fenómeno que pretende medir (validez predictiva). La diferencia en el puntaje de SNOT-20 post-tratamiento fue clínica y estadísticamente significativa, con valores mayores a 0,8 y un tamaño de efecto moderado $(0,24$ - Tabla 1). Una de las fortalezas de SNOT-20 reside en la evaluación de los 5 indicadores-objetivo. Utilizando este parámetro la sensibilidad al cambio aumenta alcanzando un tamaño de efecto casi fuerte $(0,48)$. Insistir en el uso de esta característica parece fundamental en la evaluación de efectividad de tratamientos.
Finalmente, es altamente probable que SNOT20 detecte y discrimine el fenómeno que intenta medir, con una importante área baja la curva ROC (0,895 - Figura 1$)$.

Considerando todos estos aspectos la adaptación chilena de SNOT-20 parece ser válida, confiable y de fácil aplicación. Esperamos que este instrumento sea útil en investigación y en la práctica clínica de otorrinolaringólogos, pediatras, alergólogos, inmunólogos, broncopulmonares, médicos generales y demás profesionales en estrecho y frecuente contacto con patología de la vía aérea superior.

\section{Referencias}

1. Berger W. Overview of allergic rinitis. Annals Alergy, Asthma and Inmunol. 2003; 90: 7-12.

2. Pearlman A, Conely D. Review of current guidelines related to the diagnosis and treatment of rhinosinusitis. Curr Opin Otolaryngol Head Neck Surg 2008; 16: 22630.

3. Jofre D, Pardo J, Finkelstein A. Tratamiento médico de la Rinosinusitis crónica. Rev Otorrinolaringol Cir Cabeza Cuello 2009; 69: 169-84.

4. van.Oene C, van.Reiji E, Sprangers M, Fokkens W. Quality-assessment of disease-specific quality of life questionnaires for rhinitis and rhinosinusitis: a systematic review. Allergy 2007; 62: 1359-71.

5. Caussade S, Valdivia G, Navarro H, et al. Prevalencia de síntomas de rinitis alérgica y su relación con factores de riesgo en escolares en Santiago, Chile. Rev Med Chile 2006; 134: 456-64.

6. Correa A, Salinas V. Enfermedades otorrinolaringológicas en una población infantil aparentemente sana. Rev Chil Pediatr 1987; 58(1).

7. Burt C, Schappert S. Ambulatory care visits to physician offices, hospital utpatient departments, and emergency departments: United States 1999-2000. Vital Health Stat 2004; 13: 1-70.

8. Fokkens W, Lund V, Mullol J, et al. European position paper on Nasal Polyps. Rhinology 2007; 45((20 suppl)): 1-139.

9. Bousquet J, Bullinger M, Fayol C, Marquis P, Valentin B, Burtin B. Assessment of quality of life in patients with perennial allergic rhinitis with the French version of the SF-36 Health Status Questionnarie. J Allergy Clin Inmunol 1994; 1: 182-8.

10. Wee H, Cheung Y, Li S, Fong K, Thumboo J. The impacto of diabetes mellitus and other chronic medical 
conditions on health-related Quality of Life: is the whole greater than the sum of its parts? Health Qual Life Outcomes 2005; 3: 2.

11. Metson R, Gliklich R. Clinical outcomes in patients with chronic sinusitis. Laryngoscope 2000; 110 (3 Pt 2): 24-8.

12. Meltzer E, Hamilos D, Hadley J, et al. Rhinosinusitis: Establishing definitions for clinical research and patient care. Otolaryngol Head Neck Surg 2004; 131 (6): s1-61.

13. Piccirillo J, Merrit M, Richards M. Psychometric and clinimetric validity of the 20-Item Sino-Nasal Outcome Test (SNOT-20). Otolaryngol Head Neck Surg 2002; 126: 41-7.

14. Browne J, Hopkins C, Slack R, Cano S. The Sino-Nasal Outcome Test (SNOT): Can we make it more clinically meaningful? Otolaryngol Head Neck Surg 2007; 136: 736-41.

15. Morley A, Sharp H. A review of sinonasal outcome scoring systems - which is best? Clin Otolaryngol 2005; 31: 103-9.

16. Baumann I, Blumenstock G, DeMaddalena H, Piccirillo J, Plinkert P. Quality of life in patients with chronic rhinosinusitis: validation of the Sino-Nasal Outcome Test-20 German Adapted Version. HNO 2007; 55 (1): 42-7.

17. ZUO K, Xu G, Shi J, Wen W, Fan Y. Quality of life survey on patients with chronic rhinosinusitis and nasal polyps. Zhonghua Er Bi Yan Hou Tou Jing Wai Ke Za Zhi 2006; 41 (10): 748-52.

18. Majima Y, Kurono Y, Hirakawa K, Suzaki H, Haruna S, Kawauchi $\mathrm{H}$, et al. Reliability and validity assessments of a Japanese version of QOL 20-Item Sino-Nasal Outcome Test for chronic rhinosinusitis. Auris Nasus Larynx 2010; 37: 443-8.

19. Sachanandani N, Piccirillo J, Kramper M, Thawley S, Vlahiotis A. The effect of nasally administered budesonide respules on adrenal cortex function in patients with chronic rhinosinusitis. Arch Otolaryngol Head Neck
Surg 2009; 135 (3): 303-7.

20. Ling F, Kountakis S. Rhinosinusitis task force symptoms versus the Sinonasal Outcomes Test in patients evaluated for chronic rhinosinusitis. Am J Rhinol 2007; 21: 495-8.

21. Hadley J, Mösges R, Desroisiers M, Haverstock D, van. Veenhuyzen D, Herman-Gnjidic Z. Moxifloxacion fiveday therapy versus placebo in acute bacterial rhinosinusitis. Laryngoscope 2010; 120 (5): 1057-62.

22. Wallwork B, Coman W, Mackay-Sim A, Greiff L, Cervin A. A double-blind, randomized, placebo-controlled trial of macrolide in the treatment of chronic rhinosinusitis. Laryngoscope 2006; 116: 189-93.

23. Ye J, Yu H, Draf W, Zheng C, Wang D. Technique and results of the anterior-to-posterior-to-anterior approach in revision endoscopic sinus surgery. ORL J Otorhinolaryngol Relat Spec 2009; 71 (5): 257-62.

24. Balaker A, Bergsneider M, Martin N, Wang M. Evolution of sinonasal symptoms following endoscopic anterior skull base surgery. Skull base 2010; 20 (4): 245-51.

25. McMains K, Kountakis S. Revision functional endoscopic sinus surgery: objective and subjective surgical outcomes. Am J Rhinol 2005; 19: 344-7.

26. Das S, Khichi S, Perakis H, Woodard T, Kountakis S. Effects of smoking on quality of life following sinus surgery: 4 year follow-up. Laryngoscope 2009; 119 (11): 2284-7.

27. Kelemence A, Abadoglu O, Gumus C, Berk S, Epozturk $\mathrm{K}$, Akkurt I. The frequency of chronic rhinosinusitis/ nasal polyp in COPD and its effect on the severity of COPD. COPD 2011; 8 (1): 8-12.

28. Guilermany J, Angrill J, Alobid I, Centellas S, Prades E, Roca J, et al. United airways: the impact of chronic rhinosinusitis and nasal polyps in bronchiectasic patient's quality of life. Allergy 2009; 64 (10): 1524-9.

29. Field A. Discovering Statistic using SPSS. Tercera ed. Los Angeles-Londre-Nueva Delhi-Singapore-Washington DC: SAGE; 2009. 